\title{
A Measured Method for In Situ Viscosity of Fluid in Porous Media by Nuclear Magnetic Resonance
}

\author{
Zhengming Yang $\left(\mathbb{D},{ }^{1,2}\right.$ Zhuangzhi Ma, ${ }^{1,2}$ Yutian Luo $\mathbb{D}^{1,2}$ Yapu Zhang, ${ }^{1,2}$ \\ Hekun Guo, ${ }^{1,2}$ and Wei Lin (iD) \\ ${ }^{1}$ Department of Porous Flow \& Fluid Mechanics, Research Institute of Petroleum Exploration \& Development, \\ PetroChina Company Limited, Beijing 100083, China \\ ${ }^{2}$ University of Chinese Academy of Sciences, Beijing 100493, China
}

Correspondence should be addressed to Zhengming Yang; yzhm69@petrochina.com.cn

Received 22 January 2018; Revised 11 March 2018; Accepted 26 March 2018; Published 30 April 2018

Academic Editor: Ming Zhang

Copyright (C) 2018 Zhengming Yang et al. This is an open access article distributed under the Creative Commons Attribution License, which permits unrestricted use, distribution, and reproduction in any medium, provided the original work is properly cited.

\begin{abstract}
At present, the existing measuring methods for viscosity of fluid can only obtain the viscosity of bulk fluid, while the in situ viscosity of fluid in porous media cannot be acquired. In this paper, with the combination of nuclear magnetic resonance (NMR) and physical simulation experiment, a testing method for in situ viscosity of fluid in porous media is established, and the in situ viscosity spectra of water in tight cores under different displacement conditions is obtained. The experimental results show that the in situ viscosity distribution of water in porous media is inhomogeneous, and it is not a constant but is related to the distance between water and rock walls. When the distance between fluid and rock walls is close enough (e.g., $T_{2}$ relaxation time is less than $1 \mathrm{~ms}$ ), the viscosity of fluid increases rapidly, and the in situ viscosity is greater than the bulk viscosity. Moreover, after the rock samples are saturated with water, the in situ viscosity of water is distributed as a double-peak structure. The left peak is characterized mainly by the in situ viscosity distribution of movable fluid, whose in situ viscosity is smaller, and the right peak mainly represents the in situ viscosity distribution characteristics of immovable fluid, whose in situ viscosity is larger and increases gradually. Under a relatively large driving force, the in situ viscosity amplitude of movable fluid decreases greatly, and the average in situ viscosity of residual water in the core is much higher than that of saturated water in initial state.
\end{abstract}

\section{Introduction}

Viscosity is an embodiment of physical properties for fluid [1-3]. It affects the law of subsurface fluid flow, reservoir development mode, and hydrocarbon reservoir evaluation [4-16]. Therefore, accurate measurement of fluid viscosity is of great significance. The studies indicate that the viscosity of fluid is affected mainly by the pressure, temperature, and molecular force at liquid-solid interface, and the viscosity of the same fluid behaves variously under different conditions [16-25]. At present, the influence of pressure and temperature on fluid viscosity has been much more studied [16-23]. However, little is known about the influence on the fluid viscosity of molecular force at the fluid-solid interface in porous media.

With the development of low permeability oilfields, understanding of the effect of microstructure on fluid properties in porous media becomes more and more significant, which requires people to pay more attention to the influence of the properties of porous media on fluid viscosity [24-33]. In low permeability porous media, the molecular force at the liquid-solid interface is more obvious because of its large specific surface $[24,25,30]$. Under the influence of the molecular force, liquid molecules near the liquid-solid interface will form an orderly distribution, forming a boundary layer on the liquid-solid interface. The property of the fluid in this boundary layer is different from that of the bulk fluid and its viscosity will be greater than the viscosity of the bulk fluid $[24,25]$. Marrhassing [34] found that the resin and asphaltene in crude oil were orderly distributed in pore channels. For the same crude oil sample, the viscosity of crude oil is smaller in large gaps, and the viscosity of crude oil is greater in small gaps. It was also believed that the share of the boundary layer in the small gap is larger than that in the large gap. However, 
the results of these studies still remain on the qualitative and semiquantitative description, and the viscosity distribution of the fluid in porous media cannot be quantified. On this basis, Huang et al. [24, 25] proposed a new concept of percolation fluid and the physical model of percolation fluid in porous media. It is considered that percolation fluid is a kind of fluid in porous media and includes bulk fluid and boundary fluid. Bulk fluid refers to the fluid whose properties are not affected by the interface and distributes in the middle axis of porous media channel. Accordingly, boundary fluid is a kind of fluid whose properties are influenced by the interface phenomenon, which is close to the pore wall to form a boundary layer. The formula for calculating the viscosity of percolation fluid is also given [25], but it is difficult to be applied directly because the thickness of the boundary layer and the average viscosity involved in this formula are difficult to be measured. Currently, there are many kinds of conventional viscosity testing methods, which are generally divided into three categories: capillary method $[35,36]$, rotation method [37-39], and vibration method [4043]. These methods can only measure the viscosity by direct contact with the fluid, and it is impossible to test the viscosity of the fluid in the porous media: that is, the in situ viscosity of fluid in porous media cannot be measured, whereas the in situ viscosity of the fluid in porous media is more capable of characterizing the transport capacity of the fluid, so it is especially necessary to develop the method of testing the in situ viscosity of fluid in porous media.

Viscosity measurement using nuclear magnetic resonance (NMR) is a novel viscosity test method developed in recent years, which has the advantages of noncontact and wide application range and so on and has broad application prospects in in situ viscosity measurement of fluid in rock porous media. Scholars from all over the world have done a great deal of work in studying the fluid viscosity of reservoirs by using NMR technology. In 1994, Morriss et al. [44] carried out NMR test on 31 groups of bulk crude oil. It was found that the movement of hydrogen atom was restricted with the increase of hydrogen chain, resulting in shorter relaxation time and increased viscosity. And the relationship between relaxation time and the viscosity of crude oil was also given. In 1996, Kleinberg and Vinegar [45] proposed an improved formula for measuring the viscosity of crude oil by NMR, and the temperature was introduced into the viscosity formula. In 1998, LaTorraca et al. [46] used NMR to measure the viscosity of high-viscosity crude oil. The conclusion was drawn that the geometric mean value of $T_{2}$ is more sensitive to the change of viscosity than the geometric mean value of $T_{1}$, and the meaning of the relative hydrogen index HI was put forward. In 2001, Freedman et al. [47] employed the magnetorheological fluid (MRF) method to invert the NMR data through the MRF multifluid relaxation model and studied NMR relaxation characteristics under gradient magnetic field of the sandstone, limestone, and dolomite saturated with different oil phases. Studies suggest that, under gradient magnetic field, the NMR experiment can identify different fluids in the rock pores and can also get the fluid saturation and oil-water phase viscosity and rock porosity. In 2005, Bryan et al. [4] used low-field NMR methods to

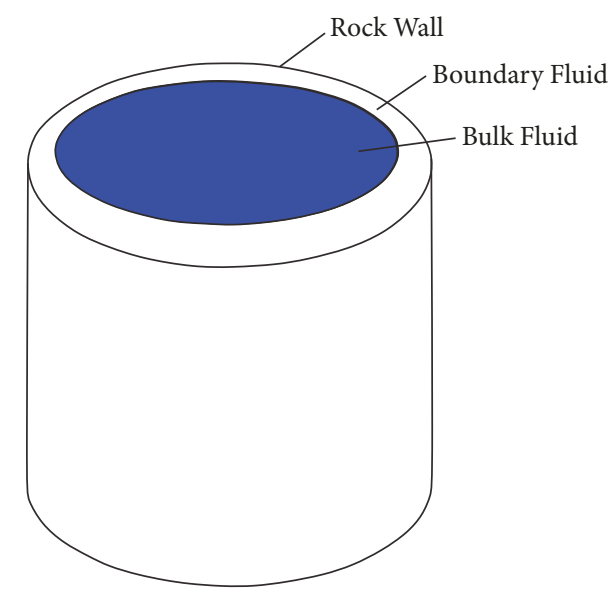

Figure 1: Physical model of percolation fluid in porous media.

measure crude oil viscosity. It was found that the higher the viscosity of the oil sample is, the lower the relative hydrogen index (RHI) is, a new NMR formula for viscosity of crude oil was put forward, and the $T_{2}$ gm and RHI of the crude oil were associated with the viscosity. In 2011, Zhang et al. [48] determined the relationship between viscosity and NMR relaxation properties through theoretical derivation and clarified the feasibility of using nuclear magnetic resonance to distinguish reservoir fluid properties. In general, at present, a relatively mature experimental method for measuring the viscosity of bulk fluid in rock pores by NMR technique has been formed, and a method for testing the viscosity of oilwater mixed phase was also initially formed. However, an effective experimental method for quantitatively measuring in situ viscosity of fluid in porous media using NMR has not yet been established.

In this paper, NMR and physical simulation experiment are combined to establish a method for in situ viscosity measurement of percolation fluid in porous media and obtain the in situ viscosity spectra of water in the tight rocks under different displacement conditions.

\section{Method and Principle}

2.1. The Nonconstant Liquid Viscosity in Rocks. Here we verify the inference that the liquid viscosity in porous media is not constant by fluid viscosity testing in different plate spacing using rheometer. According to the new concept of percolation fluid and the physical model of percolation fluid in porous media (Figure 1) proposed by Huang et al. [24, 25], we designed the viscosity experiment of the fluid at different plate spacing to verify the variation of fluid viscosity with the wall distance in the porous media. The results of constant speed mercury pressure testing of low permeability cores show that the average pore radius is generally more than $50 \mu \mathrm{m}$, and the average throat radius is generally less than $5 \mu \mathrm{m}$ [49]. Therefore, the designed plate spacing is the same as the pore and throat radius of low permeability rocks, which are $3 \mu \mathrm{m}, 4 \mu \mathrm{m}, 5 \mu \mathrm{m}, 6 \mu \mathrm{m}, 7 \mu \mathrm{m}, 8 \mu \mathrm{m}, 10 \mu \mathrm{m}$, $30 \mu \mathrm{m}, 50 \mu \mathrm{m}$, and $124 \mu \mathrm{m}$, respectively. The experimental 


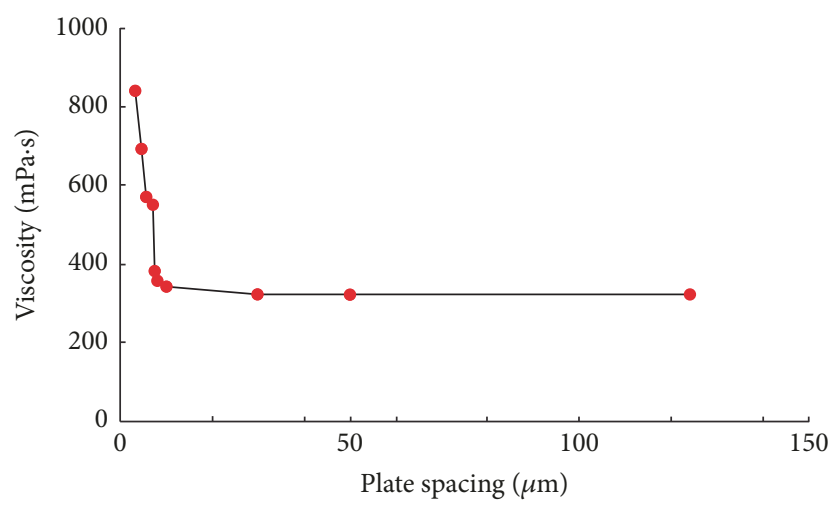

FIGURE 2: Viscosity variation of glycerol with different plate spacing.

equipment for measuring the viscosity of fluid is Anton Paar's MCR rheometer, the experimental fluid is glycerol (the viscosity in the test tube at $25^{\circ} \mathrm{C}$ is $320 \mathrm{mPa} \cdot \mathrm{s}$ ), and the test temperature is $25^{\circ} \mathrm{C}$. The experimental results are shown in Figure 2.

As can be seen from Figure 2, when the plate spacing is greater than $8 \mu \mathrm{m}$, the test viscosity of glycerol is constant and does not change with the variation of plate spacing. To the contrary, when the plate spacing is less than $8 \mu \mathrm{m}$, with the narrowing of the plate spacing, the viscosity of glycerol increases rapidly. Furthermore, when the plate spacing is reduced to $3 \mu \mathrm{m}$, the viscosity of glycerol reaches $740 \mathrm{mPa} \cdot \mathrm{s}$, which is 2.3 times bulk viscosity of glycerol.

The experiment results verify the percolation fluid model proposed by Huang et al. [24, 25] and show that, in low permeability porous media, the viscosity of the fluid is no longer a constant. There is a boundary fluid near the solid wall, whose viscosity is much larger than that of the bulk fluid. Therefore, in the low permeability porous media, the in situ viscosity of the fluid is larger than that measured in the laboratory. Hence, it is so necessary to study the in situ viscosity of fluid in porous media, in order to help us understand the actual viscosity of the fluid in the ground.

2.2. Testing Principle for Fluid Viscosity by NMR. The principle of NMR is to measure the signal of hydrogen atoms in the magnetic field. There is a certain amount of hydrogen atoms in the oil and water, and when a magnetic pulse signal is applied, these hydrogen atoms will produce a strong attenuation response $[45,46]$. If NMR is performed on oil-bearing or water-bearing rocks, due to the absence of paramagnetic elements in the rocks, there is no signal response in the rocks, and the measured signals are only from the oil and water in the rock pores [47]. In nuclear magnetic resonance experiments, hydrogen atoms are polarized by the external magnetic field, and the energy acts on hydrogen atoms in the form of radio frequency pulses, causing the hydrogen atoms to absorb energy and undergo energy level transitions. After the hydrogen atom absorbs energy, it transfers energy to other rotating atoms or solid surfaces to restore to the original state of equilibrium [4]. Because the measurement time of the longitudinal attenuation signal is longer and is easily

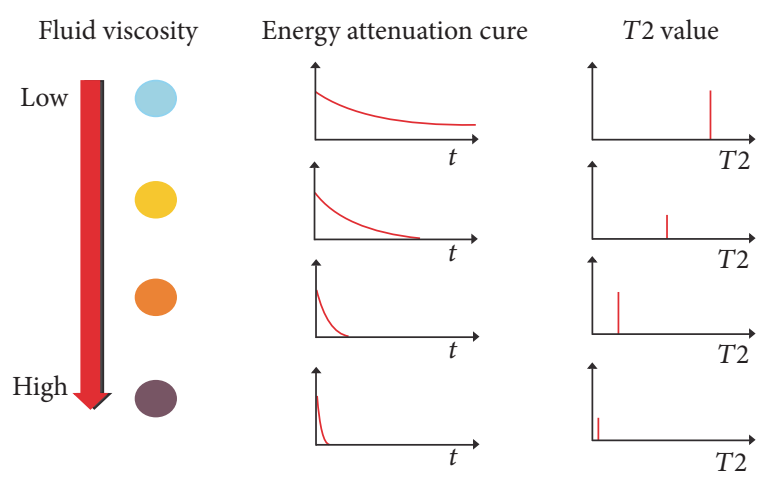

FIGURE 3: Relationship between fluid viscosity and energy attenuation rate and $T_{2}$ value.

affected by the external environment, the NMR measurement signal is a transverse attenuation signal. The attenuation signals can be converted to the attenuation curve, which is the superposition of the attenuation signals of different fluid phases. The fluid components are different, so the attenuation signals and the attenuation curves are different, and the attenuation curve functions conform to the single index or multiexponential function [48-51].

Eyring viscosity theory shows $[50,51]$ that the viscosity is related to the diffusion conductivity. Even if fluid does not flow, its viscosity can also be measured, and NMR technology can be used to measure the diffusion conduction characteristics of fluids. NMR can be used to measure the attenuation response of liquid hydrogen atom in magnetic field under the action of external magnetic field. Prammer [52] presented the relation between the amplitude attenuation of NMR and the transverse relaxation time $\left(T_{2}\right)$ and pointed out that the fluid viscosity is correlated positively with the attenuation rate of fluid amplitude. The smaller the fluid viscosity is, the slower the amplitude attenuation rate is, and the greater the relaxation time $T_{2}$ is. Instead, the larger the fluid viscosity is, the faster the amplitude attenuation rate is, and the smaller the relaxation time $T_{2}$ is. Its principle is shown in Figure 3 and (1).

The equation of energy attenuation curve in magnetic field is as follows [52]:

$$
M(t)=M_{0} e^{-t / T_{2}},
$$

where $M(t)$ is the macroscopic magnetization at moment $t$, $M_{0}$ is the equilibrium magnetization, $T_{2}$ is the transverse relaxation time, and $t$ is time, $\mathrm{ms}$.

From the energy attenuation curve equation, it can be seen as follows: the greater the $T_{2}$ value is, the smoother the energy attenuation curve is. The smaller the $T_{2}$ value is, the faster the energy attenuation curve falls. The transverse relaxation time $T_{2}$ is related to the properties of fluid itself and the interaction between fluid and rock pore surface, and so on. Therefore, NMR $T_{2}$ value can be used to characterize the viscosity of fluid.

2.3. Relationship between Fluid Viscosity and $T_{2}$ Peak. The purpose of this section is to find the empirical formula of 


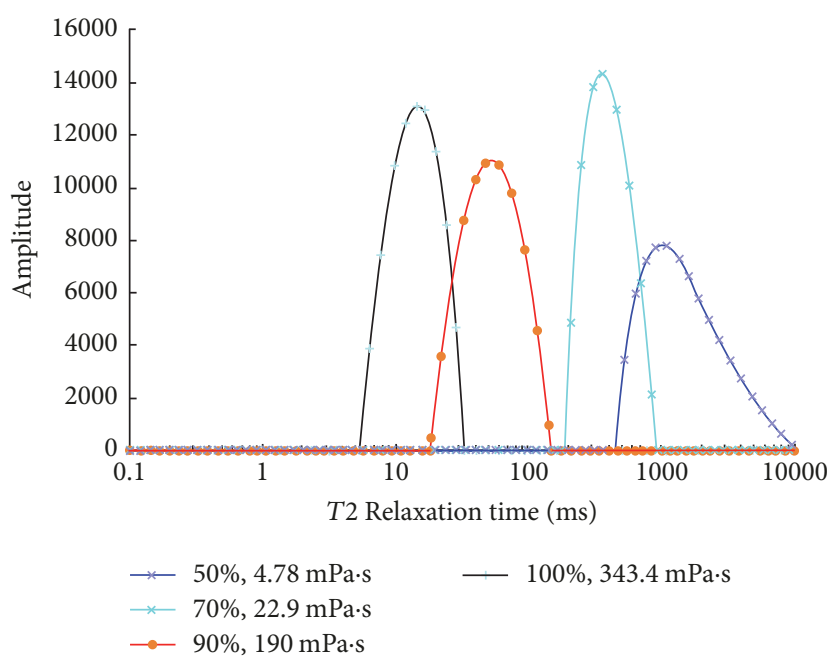

(a) Glycerol

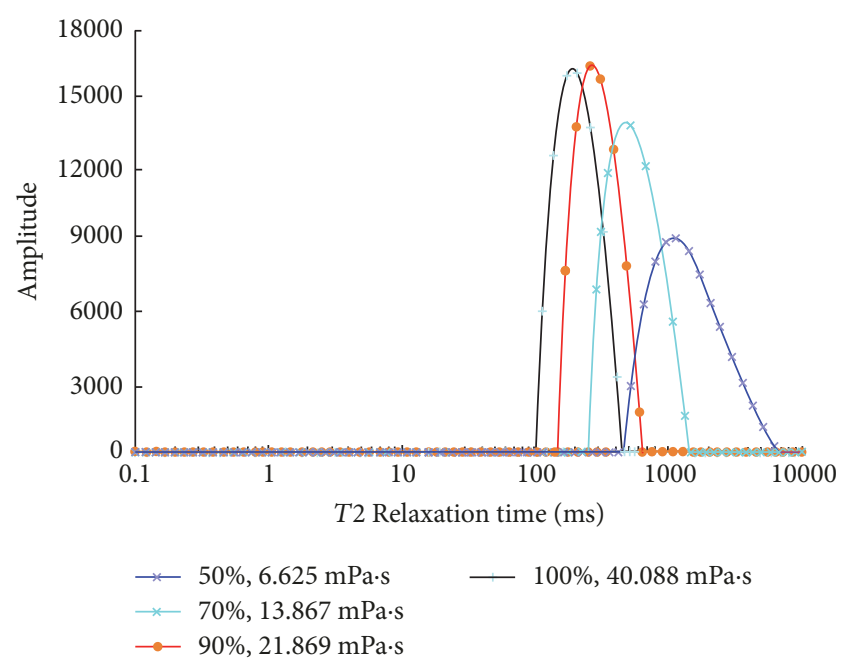

(b) Polyethylene glycol

FIgURE 4: $T_{2}$ spectra of solutions at different concentrations.

fluid viscosity and NMR $T_{2}$ values by conducting NMR experiments on different kinds of fluids with different viscosity, providing a basis for testing in situ fluid viscosity in porous media.

The experimental instrument is the RecCore4 NMR equipment developed independently by China Petroleum Exploration and Development Research Institute Langfang Branch. Its operating frequency is $2 \mathrm{MHz}$, the echo time $T_{E}$ is adjustable, and the minimum time is $2 \mu \mathrm{s}$. Glycerol and polyethylene glycol are selected as test fluid, prepared into experimental solutions with different concentrations $(10 \%$ $90 \%$ ), and placed in the test tubes.

The experimental steps are as follows: first, Ubbelohde viscometer is used to measure the viscosity of different concentration solutions, and then the NMR spectra of different concentration solutions are obtained with the RecCore 4 NMR equipment; finally, we analyze and process the data to get the experimental results shown in Figures 3 and 4 .

It can be seen from Figures 4 and 5 that there is a good correlation between viscosity and $T_{2}$ peak value $\left(T_{2 f}\right)$. The relationship is as follows:

$$
\begin{aligned}
\mu & =23938 T_{2 f}^{-1.236}, \\
R^{2} & =0.983 .
\end{aligned}
$$

Equation (2) indicates that the greater the $T_{2}$ peak value is, the smaller the fluid viscosity is. Contrarily, the smaller the $T_{2}$ peak value is, the greater the fluid viscosity is.

The above experimental results demonstrate that NMR method can be used to test the viscosity of different liquid. The relationship between fluid viscosity and $T_{2}$ peak of NMR is clearly defined as follows:

$$
\mu=\frac{a}{T_{2 f}^{b}},
$$

where $\mu$ is the viscosity, $T_{2 f}$ is the $T_{2}$ peak, and $a$ and $b$ are the parameters related to the properties of the solution.

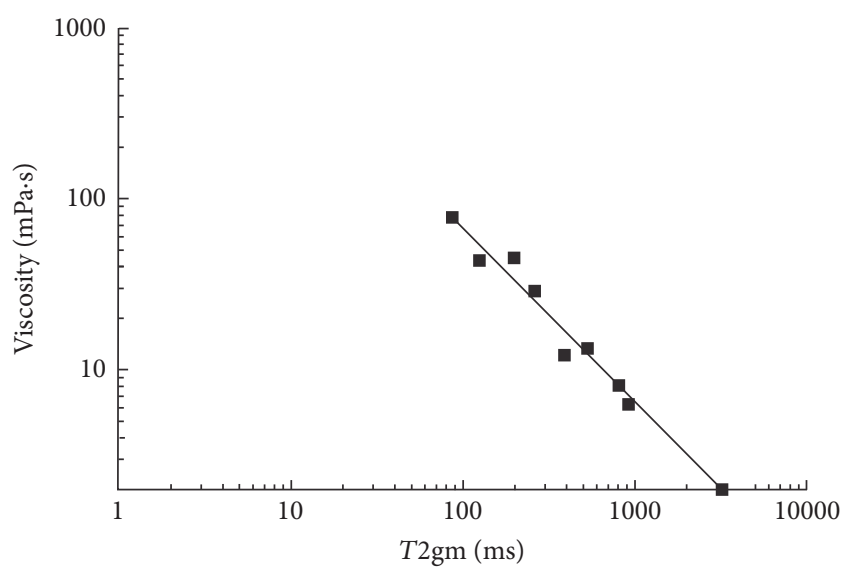

FIgURE 5: Relationship between fluid viscosity and $T_{2}$ relaxation time.

\section{In Situ Viscosity Measurement of Fluid in Porous Media}

Taking the water in porous media as an example, the in situ viscosity of fluid in porous media is measured here. The $T_{2}$ relaxation spectrum of water in rock sample can be obtained by NMR technology. By (3), the $T_{2}$ spectra of fluid can be converted into the in situ viscosity spectra of fluid in different pores and throats of porous media.

In order to test the in situ viscosity of fluid in porous media, five tight cores in Changqing and Jilin were selected, and their petrophysical parameters are shown in Table 1 . The experimental steps are as follows.

Firstly, the above five cores were completely saturated with water; at the same time, the NMR spectra were tested. Then, different centrifugal experiments of $0.145 \mathrm{MPa}$, 0.290 MPa, 0.717 MPa, 1.441 MPa, 2.069 MPa, and 2.876 MPa 
TABLE 1: Petrophysical parameters of 5 tight rock cores.

\begin{tabular}{lccccc}
\hline Core number & Oil block & Length $(\mathrm{cm})$ & Diameter $(\mathrm{cm})$ & $\begin{array}{c}\text { Gas } \\
\text { measurement } \\
\text { porosity }(\%)\end{array}$ & $\begin{array}{c}\text { Gas } \\
\text { measurement } \\
\text { permeability } \\
(\mathrm{mD})\end{array}$ \\
\hline 1 & Jilin & 2.664 & 2.438 & 8.08 & 0.130 \\
2 & & 3.236 & 2.503 & 9.42 & 0.673 \\
\hline 3 & Changqing & 3.225 & 2.514 & 12.30 & 0.058 \\
4 & & 3.076 & 2.515 & 10.30 & 0.116 \\
\hline
\end{tabular}

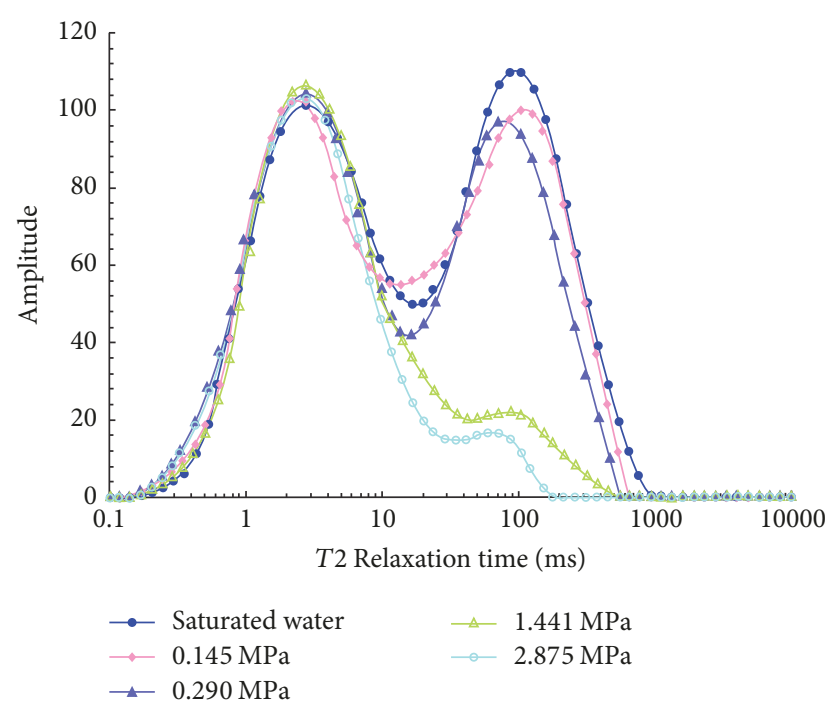

FIGURE 6: $T_{2}$ spectra of $0.130 \mathrm{mD}$ rock core after centrifugation with different centrifugal pressures.

were carried out for each core (different centrifugal pressures represent different displacement pressures), and NMR $T_{2}$ spectra under each centrifugal pressure were captured. Finally, the experimental data was analyzed and processed to obtain the experimental results as shown in Figures 6-9.

\section{Results and Discussion}

Figure 6 is a NMR spectrum of $0.130 \mathrm{mD}$ rock sample in Jilin oilfield. When the centrifugal pressure is $0.145 \mathrm{MPa}$ and $0.290 \mathrm{MPa}$, the yield of water centrifuged out of rock core is only $4.49 \%$ and $7.38 \%$, respectively. When the centrifugal pressure increases to $1.441 \mathrm{MPa}$, the yield of water centrifuged out of rock core is $41.18 \%$. However, when the centrifugal pressure is increased from $1.441 \mathrm{MPa}$ to $2.876 \mathrm{MPa}$, the production increment of water centrifuged out of rock core is only $5.13 \%$.

According to (3), NMR spectra of $0.130 \mathrm{mD}$ tight rock sample under saturated water condition in Figure 6 are treated, and we obtain the relationship of in situ viscosity and $T_{2}$ relaxation time in porous media under saturated water condition (Figure 7). Studies show that [45] $T_{2}$ relaxation time can represent the distance between fluid and rock wall. The

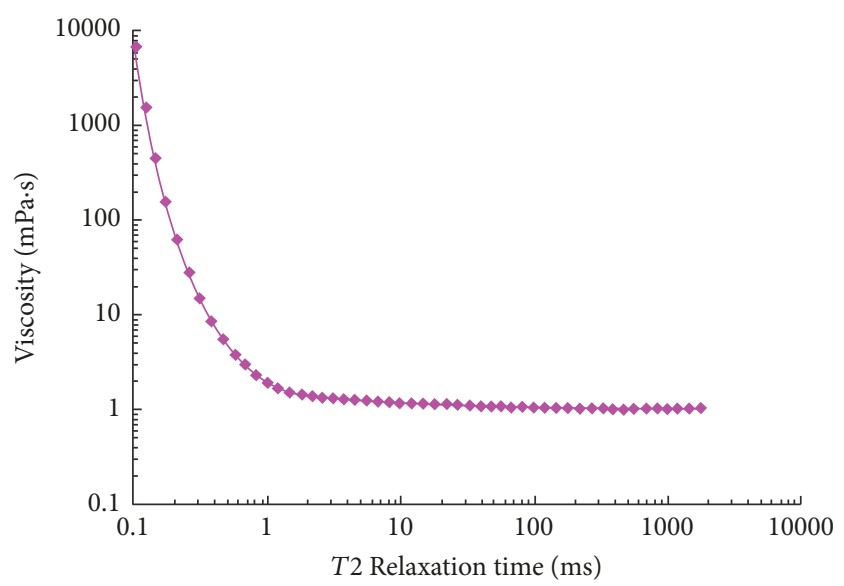

FIGURE 7: Relationship between in situ viscosity and $T_{2}$ relaxation time of $0.130 \mathrm{mD}$ rock core after saturated water.

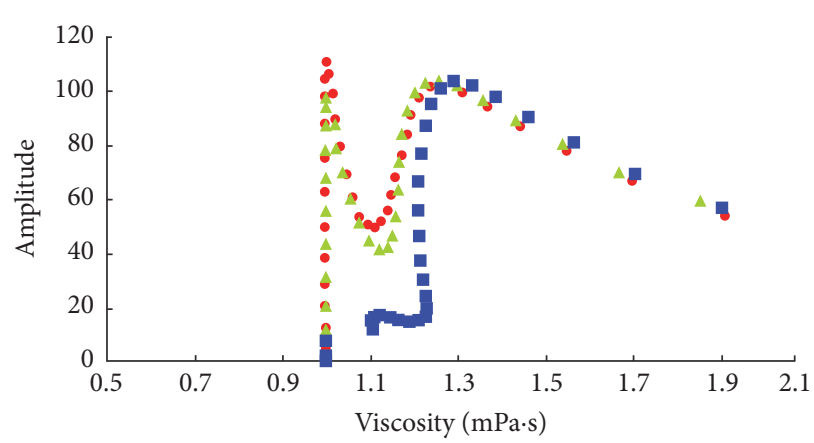

$$
\begin{aligned}
& \text { - Saturated water } \\
& \text { - } 0.290 \mathrm{MPa} \\
& \text { - } 2.876 \mathrm{MPa}
\end{aligned}
$$

FIGURE 8: In situ viscosity spectrum of $0.130 \mathrm{mD}$ rock sample under different displacement conditions.

larger the $T_{2}$ relaxation time, the greater the distance between the fluid and the rock wall. So it can be found from Figure 7 that the in situ viscosity of water in porous media is not a constant, and it is related to the distance between water and rock wall. When water is closer to wall, its in situ viscosity is greater than its bulk fluid viscosity. What is more, when $T_{2}$ relaxation time is less than $1 \mathrm{~ms}$, that is, the closer the fluid is to rock wall, the higher the in situ viscosity is. At the 


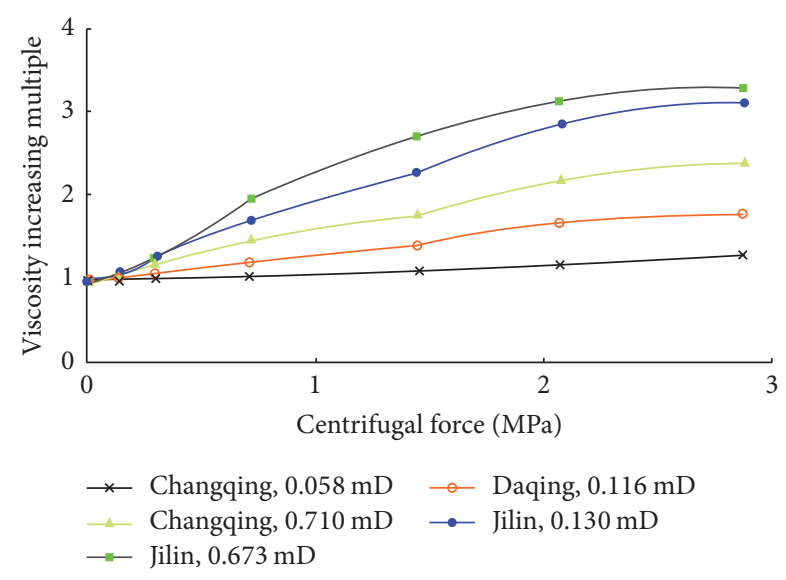

FIGURE 9: Variation of average in situ viscosity of water in tight rock under different centrifugal pressures.

same time, this result also indicates that the interface force between fluid and rock is stronger, and it is extremely difficult to develop the fluid with $T_{2}$ time less than $1 \mathrm{~ms}$.

Figure 8 shows the in situ viscosity spectra of $0.130 \mathrm{mD}$ rock samples under different displacement conditions. We realize from Figure 8 that the in situ viscosity of fluid is distributed unevenly in porous media. Under the saturated water state of rock sample, the in situ viscosity spectrum of water presents a double-peak structure. For the left peak of in situ viscosity spectrum, the viscosity of fluid is smaller, which mainly characterizes the in situ viscosity distribution characteristics of movable fluid at the right peak of NMR $T_{2}$ spectrum. This part of fluid is distributed mainly in the middle of pores and throats. For the right peak of in situ viscosity spectrum, the viscosity increases greatly, which mainly characterizes the in situ viscosity distribution characteristics of nonmovable fluid at the left peak of NMR $T_{2}$ spectrum. This part of fluid is distributed mainly in the boundary layer of pores and throats or nearby the wall. Furthermore, as the in situ viscosity is greater than $1.3 \mathrm{mPa} \cdot \mathrm{s}$, the proportion of nonmovable fluid decreases sharply with the increase of viscosity. At a relatively lower driving force (e.g., $0.290 \mathrm{MPa}$ ), the in situ viscosity amplitude of movable fluid decreases smaller, and the in situ viscosity amplitude of nonmovable fluid coincides basically with the curve under the saturated water condition. This indicates that only some movable fluid can be exploited in tight cores under the small pressure difference, but the boundary fluid cannot be developed basically. Under the larger driving force (e.g., 2.876 MPa), the in situ viscosity amplitude of movable fluid drops greatly, which indicates the movable fluid is produced more. However, the fluid with the viscosity more than $1.3 \mathrm{mPa} \cdot \mathrm{s}$ is harvested less, which indicates that it is very difficult to exploit the boundary fluid. Hence, findings also include that the production degree of fluid in tight rock is lower under a small pressure gradient. Only under a high pressure gradient, the production degree of fluid is relatively higher.
Figure 9 shows the variation of the average in situ viscosity of residual water in rock cores with different permeability under different centrifugal pressures. The longitudinal coordinate is the increasing multiple of viscosity, which is the ratio of the average in situ viscosity of the residual water in rock cores and the average in situ viscosity of the water in rock cores with saturated water state under a centrifugal pressure.

It can be seen from Figure 9 that the increasing multiple of viscosity in rock core is small under relatively low centrifugal pressure (e.g., $0.145 \mathrm{MPa}$ and $0.290 \mathrm{MPa}$ ). This is because the amount of yield water centrifuged out of rock core under a smaller centrifugal pressure is small, and large amounts of water still exist in the large pores, which has little influence on the average in situ viscosity of water in rock core. Only when the centrifugal pressure increases to a certain value (e.g., 0.717 MPa, 1.441 MPa, and 2.069 MPa), the increasing multiple of viscosity in rock core increases rapidly. This is because that, with the centrifugal pressure increases, the amount of water centrifuged out of rock core increases, and the production of water in the large channel increases, the proportion of the boundary fluid thus rises, which has great influence on the average in situ viscosity of water in rock core. When the centrifugal pressure is once increased from $2.069 \mathrm{MPa}$ to $2.876 \mathrm{MPa}$, the increasing multiple of viscosity in core increases slowly, because the amount of water produced is less, which has a little effect on the average in situ viscosity of the water in rock core. For rock samples in the same block (Changqing or Jilin), under the same centrifugal pressure, the viscosity increasing multiple of water in rock increases with the increase of permeability. This phenomenon can be interpreted as, under the same centrifugal pressure, the higher the permeability, the more the amount of water centrifuged out of rock core. Under the same centrifugal pressure, the viscosity increasing multiple of water in Jilin is higher than that in Changqing, which can be ascribed to the different reservoir characteristics of Changqing and Jilin tight cores reported by Yang et al. [49].

\section{Conclusions}

An approach to test in situ viscosity of fluid in porous media by NMR is reported in this work. Studies have shown that there is a better correlation between fluid viscosity and $T_{2}$ peak value and the in situ viscosity of fluid is distributed unevenly in porous media. In saturated water state, the in situ viscosity of water in rock samples is distributed as a doublepeak structure. Moreover, for the tight rocks with saturated water, after a small amount of water is centrifuged, the average in situ viscosity of water in rocks has little difference with that of initial status. However, when more water is separated from the rocks, the average in situ viscosity of water in rocks is significantly higher than that of initial status. Furthermore, under the same centrifugal pressure, the ratio of the average in situ viscosity of water in rocks to that of initial status increases with the increase of permeability, and the average in situ viscosity increasing multiple of water in Jilin is higher than that in Changqing, which is due to the different reservoir characteristics of Changqing and Jilin tight rocks. The paper first proposes the method for measuring the in situ viscosity 
of fluid in porous media, which can provide guidance for the development of movable oil in oil field. At the same time, the accuracy and application range of the method will be improved after a large number of tests of different fluids.

\section{Conflicts of Interest}

The authors declare that they have no conflicts of interest.

\section{Acknowledgments}

The authors gratefully acknowledge financial support from the National Science and Technology Major Project (2017ZX05013-001).

\section{References}

[1] A. Roszkowski, M. Bogdan, W. Skoczynski, and B. Marek, "Testing viscosity of MR fluid in magnetic field," Measurement Science Review, vol. 8, no. 3, pp. 58-60, 2008.

[2] T. Schäfer, "Fluid dynamics and viscosity in strongly correlated fluids," Annual Review of Nuclear and Particle Science, vol. 64, no. 1, pp. 125-148, 2014.

[3] D. Bonn, H. Kellay, M. Bräunlich, M. B. Amar, and J. Meunier, "Viscous fingering in complex fluids," Physica A: Statistical Mechanics and its Applications, vol. 220, no. 1-2, pp. 60-73, 1995.

[4] J. Bryan, A. Kantzas, and C. Bellehumeur, "Oil-viscosity predictions from low-field NMR measurements," SPE Reservoir Evaluation and Engineering, vol. 8, no. 1, pp. 44-52, 2005.

[5] H. Singh, F. Javadpour, A. Ettehadtavakkol, and H. Darabi, "Nonempirical apparent permeability of shale," SPE Reservoir Evaluation and Engineering, vol. 17, no. 3, pp. 414-424, 2014.

[6] H. Singh and F. Javadpour, "Langmuir slip-Langmuir sorption permeability model of shale," Fuel, vol. 164, pp. 28-37, 2016.

[7] H. Singh and J. Cai, "Screening improved recovery methods in tight-oil formations by injecting and producing through fractures," International Journal of Heat and Mass Transfer, vol. 116, pp. 977-993, 2018.

[8] H. Singh, "A critical review of water uptake by shales," Journal of Natural Gas Science and Engineering, vol. 34, pp. 751-766, 2016.

[9] R. Song, M. Cui, and J. Liu, "Single and multiple objective optimization of a natural gas liquefaction process," Energy, vol. 124, pp. 19-28, 2017.

[10] A. F. Li Petroleum University Press, Dongying, China, 2011.

[11] W. Lin, Z. Yang, X. Li et al., "A method to select representative rock samples for digital core modeling," Fractals. Complex Geometry, Patterns, and Scaling in Nature and Society, vol. 25, no. 4, Article ID 1740013, 1740013, 9 pages, 2017.

[12] R. Song, M. Cui, and J. Liu, "A correlation for heat transfer and flow friction characteristics of the offset strip fin heat exchanger," International Journal of Heat and Mass Transfer, vol. 115, pp. 695-705, 2017.

[13] W. Lin, X. Li, Z. Yang et al., "A new improved threshold segmentation method for scanning images of reservoir rocks considering pore fractal characteristics," Fractals, vol. 26, article 1840003, no. 2, 2018.

[14] R. Song, J. Liu, and M. Cui, "Single- and two-phase flow simulation based on equivalent pore network extracted from micro-CT images of sandstone core," SpringerPlus, vol. 5, no. 1, article no. 817, 2016.
[15] M. Zhang, Y. Yin, and B. Huang, "Mechanisms of rainfallinduced landslides in gently inclined red beds in the eastern Sichuan Basin, SW China," Landslides , vol. 12, no. 5, pp. 973983, 2015.

[16] W. Lin, Z. Yang, J. Wang, T. Chen, and X. Shi, "Wormlike micelles with $\mathrm{pH}$-induced rheological property formed by cationic surfactant/anthranilic acid mixed aqueous solution," Journal of Molecular Liquids, vol. 224, pp. 333-337, 2016.

[17] W. Wei, J. Cai, X. Hu et al., "A numerical study on fractal dimensions of current streamlines in two-dimensional and threedimensional pore fractal models of porous media," Fractals, vol. 23, no. 1, Article ID 1540012, 2015.

[18] M. Zhang and M. J. McSaveney, "Is air pollution causing landslides in China?" Earth and Planetary Science Letters, vol. 481, pp. 284-289, 2018.

[19] J. Wang, S. Wang, W. Lin, Z. Kang, and Q. You, "Formula optimization and rheology study of clean fracturing fluid," Journal of Molecular Liquids, vol. 241, pp. 563-569, 2017.

[20] T. Watanabe and A. Higuchi, "A new apparatus for measuring elastic wave velocity and electrical conductivity of fluidsaturated rocks at various confining and pore-fluid pressures," Geofluids, vol. 14, no. 3, pp. 372-378, 2014.

[21] D. Zhu, Q. Meng, Z. Jin, and W. Hu, "Fluid environment for preservation of pore spaces in a deep dolomite reservoir," Geofluids, vol. 15, no. 4, pp. 527-545, 2015.

[22] C. Huber and Y. Su, "A pore-scale investigation of the dynamic response of saturated porous media to transient stresses," Geofluids, vol. 15, no. 1-2, pp. 11-23, 2015.

[23] R. Song, M. Cui, J. Liu, P. G. Ranjith, and Y. Lei, "A Pore-Scale Simulation on Thermal-Hydromechanical Coupling Mechanism of Rock," Geofluids, vol. 2017, pp. 1-12, 2017.

[24] Y. Z. Huang, Percolating Flow Mechanism of Low Permeability Reservoir, Petroleum Industry Press, Beijing, China, 1998.

[25] Y. Huang, Z. Yang, Y. He, and X. Wang, "An overview on nonlinear porous flow in low permeability porous media," Theoretical \& Applied Mechanics Letters, vol. 3, no. 2, p. 022001, 2013.

[26] G. J. Hirasaki and J. B. Lawson, "Mechanisms of foam flow in porous media: apparent viscosity in smooth capillaries," SPE Journal, vol. 25, no. 2, pp. 176-190, 1985.

[27] Y. Xia, J. Cai, W. Wei et al., "A new method for calculating fractal dimensions of porous media based on pore size distribution," Fractals, vol. 26, article 1850006, no. 1, 2018.

[28] Y.-L. Feng, Y. Zhang, B.-Y. Ji, and W.-Z. Mu, "Micro-acting force in boundary layer in low-permeability porous media," Chinese Physics Letters, vol. 28, no. 2, Article ID 024703, 2011.

[29] J. C. Cai and B. M. Yu, "A discussion of the effect of tortuosity on the capillary imbibition in porous media," Transport in Porous Media, vol. 89, no. 2, pp. 251-263, 2011.

[30] W. Lin, X. Li, Z. Yang et al., "Construction of Dual Pore 3-D Digital Cores with a Hybrid Method Combined with Physical Experiment Method and Numerical Reconstruction Method," Transport in Porous Media, vol. 120, no. 1, pp. 227-238, 2017.

[31] J. G. Celauro, V. A. Torrealba, Z. T. Karpyn, K. A. Klise, and S. A. Mckenna, "Pore-scale multiphase flow experiments in bead packs of variable wettability," Geofluids, vol. 14, no. 1, pp. 95-105, 2014.

[32] J. Liu, M. Wu, Z. Zhu, and Z. Shao, "A study on the mechanical properties of the representative volume element in fractal porous media," Geofluids, vol. 2017, Article ID 7905218, 10 pages, 2017. 
[33] R. Song, J. Liu, and M. Cui, "A new method to reconstruct structured mesh model from micro-computed tomography images of porous media and its application," International Journal of Heat and Mass Transfer, vol. 109, pp. 705-715, 2017.

[34] H. Marrhassing, Mechanism of petrophysics and petrochemical, Petroleum Industry Press, Beijing, China, 1987.

[35] E. Hatschek, The Viscosity of Liquids, D. Van Nostrand Co., New York, NY, USA, 1963.

[36] X. Y. Liu, M. G. He, and Y. Zhang, "Viscosity of water in the region around the critical point," The Journal of Supercritical Fluids, vol. 63, pp. 150-154, 2012.

[37] A. Colafigli, L. Mazzei, P. Lettieri, and L. Gibilaro, "Apparent viscosity measurements in a homogeneous gas-fluidized bed," Chemical Engineering Science, vol. 64, no. 1, pp. 144-152, 2009.

[38] L. A. Belfiore, "Dynamic shear in continuous-flow rotating-disk catalytic reactors with stress-sensitive kinetics based on Curie's theorem in non-equilibrium thermodynamics," Chemical Engineering Science, vol. 65, no. 2, pp. 680-691, 2010.

[39] M. Iguchi, Y. Hiraga, K. Kasuya et al., "Viscosity and density of poly(ethylene glycol) and its solution with carbon dioxide at $353.2 \mathrm{~K}$ and $373.2 \mathrm{~K}$ at pressures up to $15 \mathrm{MPa}$," The Journal of Supercritical Fluids, vol. 97, pp. 63-73, 2015.

[40] M. J. Assael, C. P. Oliveira, M. Papadaki, and W. A. Wakeham, "Vibrating-wire viscometers for liquids at high pressures," International Journal of Thermophysics, vol. 13, no. 4, pp. 593615, 1992.

[41] X. Meng, P. Zheng, J. Wu, and Z. Liu, "Density and viscosity measurements of diethyl adipate from (303 to 373) $\mathrm{K}$ and up to $20 \mathrm{MPa}, "$ Journal of Chemical \& Engineering Data, vol. 53, no. 7, pp. 1474-1478, 2008.

[42] J. C. F. Diogo, F. J. P. Caetano, and J. M. N. A. Fareleira, "Viscosity and density measurements of compressed liquid dimethyl adipate using oscillating body techniques," Fluid Phase Equilibria, vol. 367, pp. 85-94, 2014.

[43] S. V. D. Freitas, J. J. Segovia, M. Carmen Martín et al., "Measurement and prediction of high-pressure viscosities of biodiesel fuels," Fuel, vol. 122, pp. 223-228, 2014.

[44] C. E. Morriss, R. Freedman, C. Straley, M. Johnston, H. J. Vinegar, and P. N. Tutunjian, "Hydrocarbon saturation and viscosity estimation from NMR logging in the Belridge diatomite," Log Analyst, vol. 38, no. 2, pp. 44-59, 1997.

[45] R. L. Kleinberg and H. J. Vinegar, "NMR properties of reservoir fluids," Log Analyst, vol. 37, no. 6, pp. 20-32, 1996.

[46] G. A. LaTorraca, K. J. Dunn, P. R. Webber, and R. M. Carlson, "Low-field NMR determinations of the properties of heavy oils and water- in-oil emulsions," Magnetic Resonance Imaging, vol. 16, no. 5-6, pp. 659-662, 1998.

[47] R. Freedman, N. Heaton, and M. Flaum, "Field applications of a new nuclear magnetic resonance fluid characterization method," SPE Reservoir Evaluation and Engineering, vol. 5, no. 6, pp. 455-464, 2002.

[48] Z. P. Zhang, P. J. Li, and D. P. Jiang, "Study on NMR fluid identification method," nner Mongolia Petrochemical Industry, vol. 37, no. 3, pp. 29-30, 2011.

[49] Z. M. Yang, H. K. Guo, and X. W. Liu, Characteristic Experimental Technology of an Extra-Ultra Low Permeability Reservoir, Petroleum Industry Press, Beijing, China, 2012.

[50] H. Eyring, "Viscosity, plasticity, and diffusion as examples of absolute reaction rates," The Journal of Chemical Physics, vol. 4, no. 4, pp. 283-291, 1936.
[51] Z. Liu, Y. Yang, J. Yao, Q. Zhang, J. Ma, and Q. Qian, "Pore-scale remaining oil distribution under different pore volume water injection based on CT technology," ADvances in Geo-Energy Research, vol. 1, no. 3, pp. 171-181, 2017.

[52] M. G. Prammer, "NMR logging-while-drilling (1995-2000)," Concepts in Magnetic Resonance, vol. 13, no. 6, pp. 409-411, 2001. 

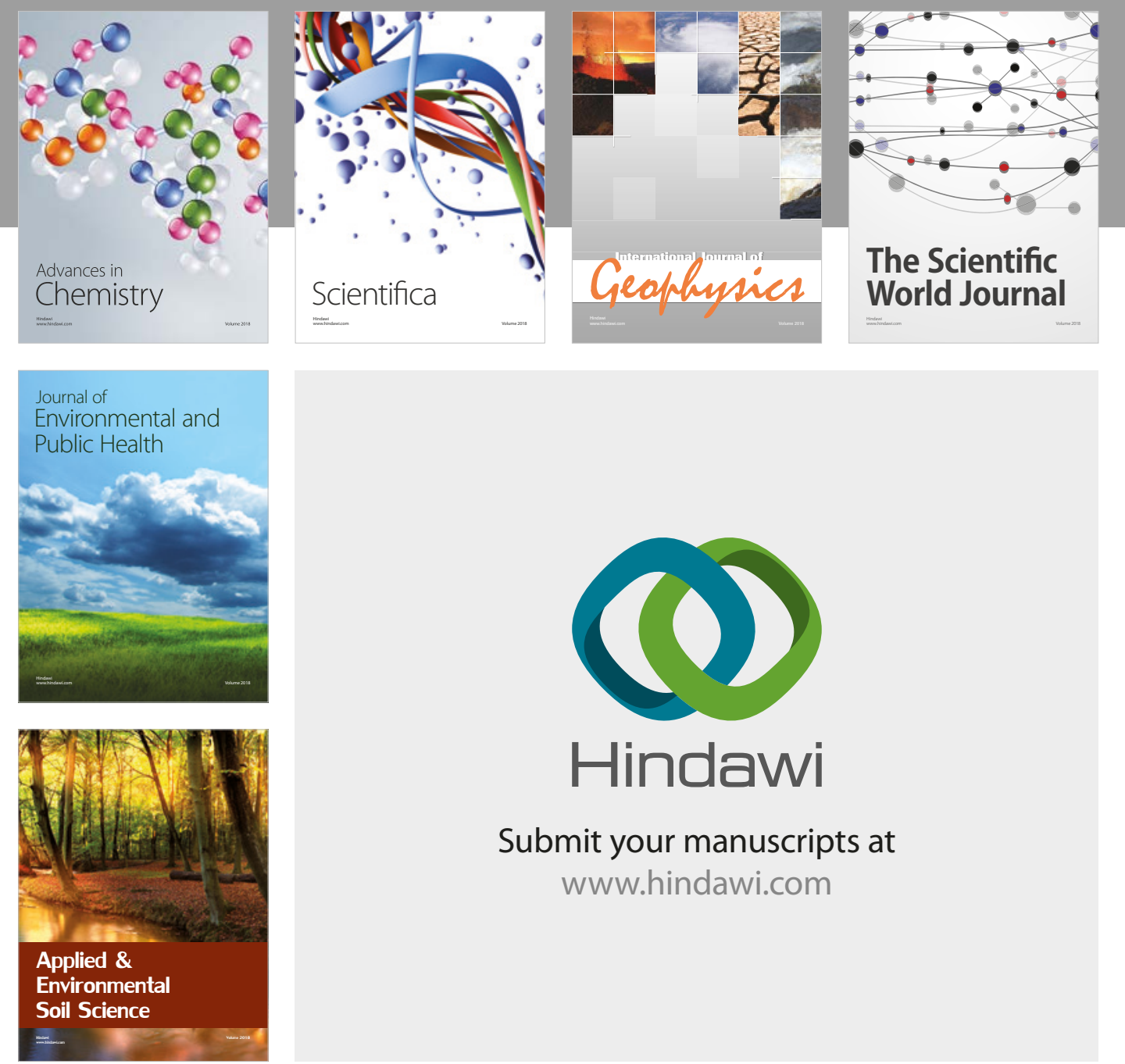

The Scientific

\section{World Journal}
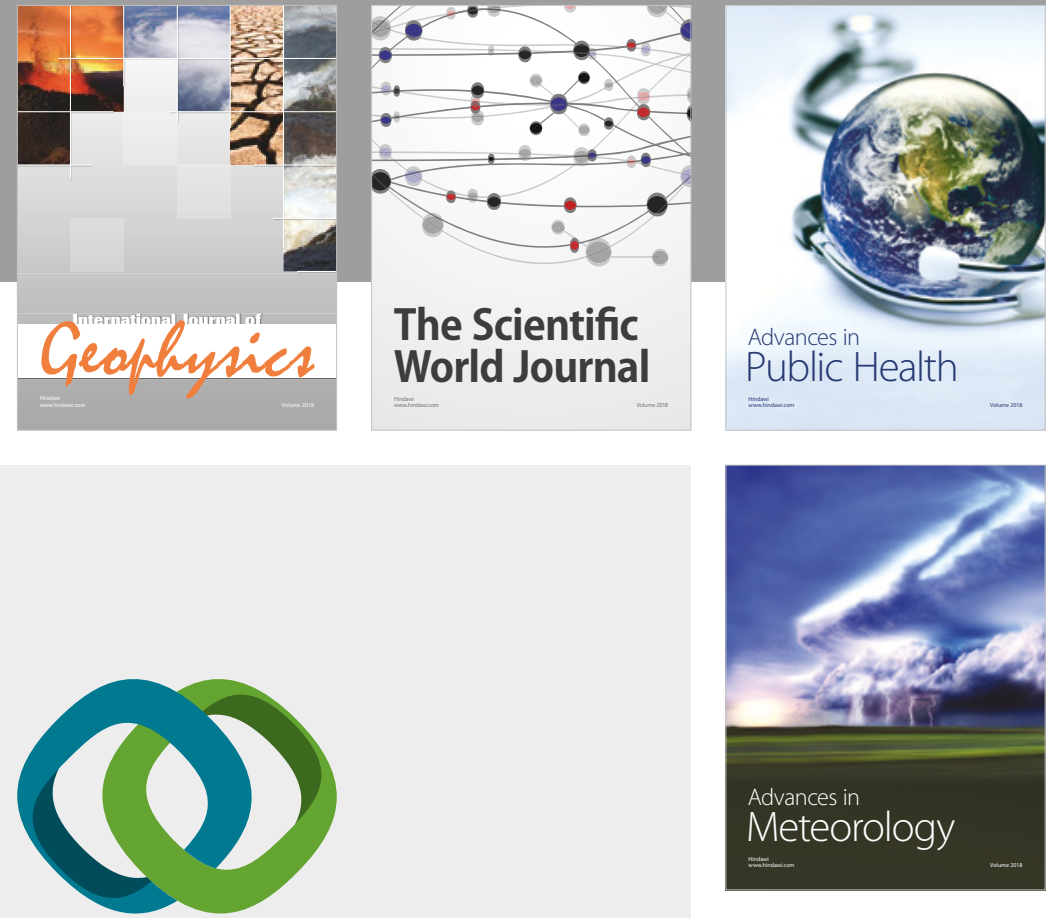

Advan

Public Health

\section{Hindawi}

Submit your manuscripts at

www.hindawi.com
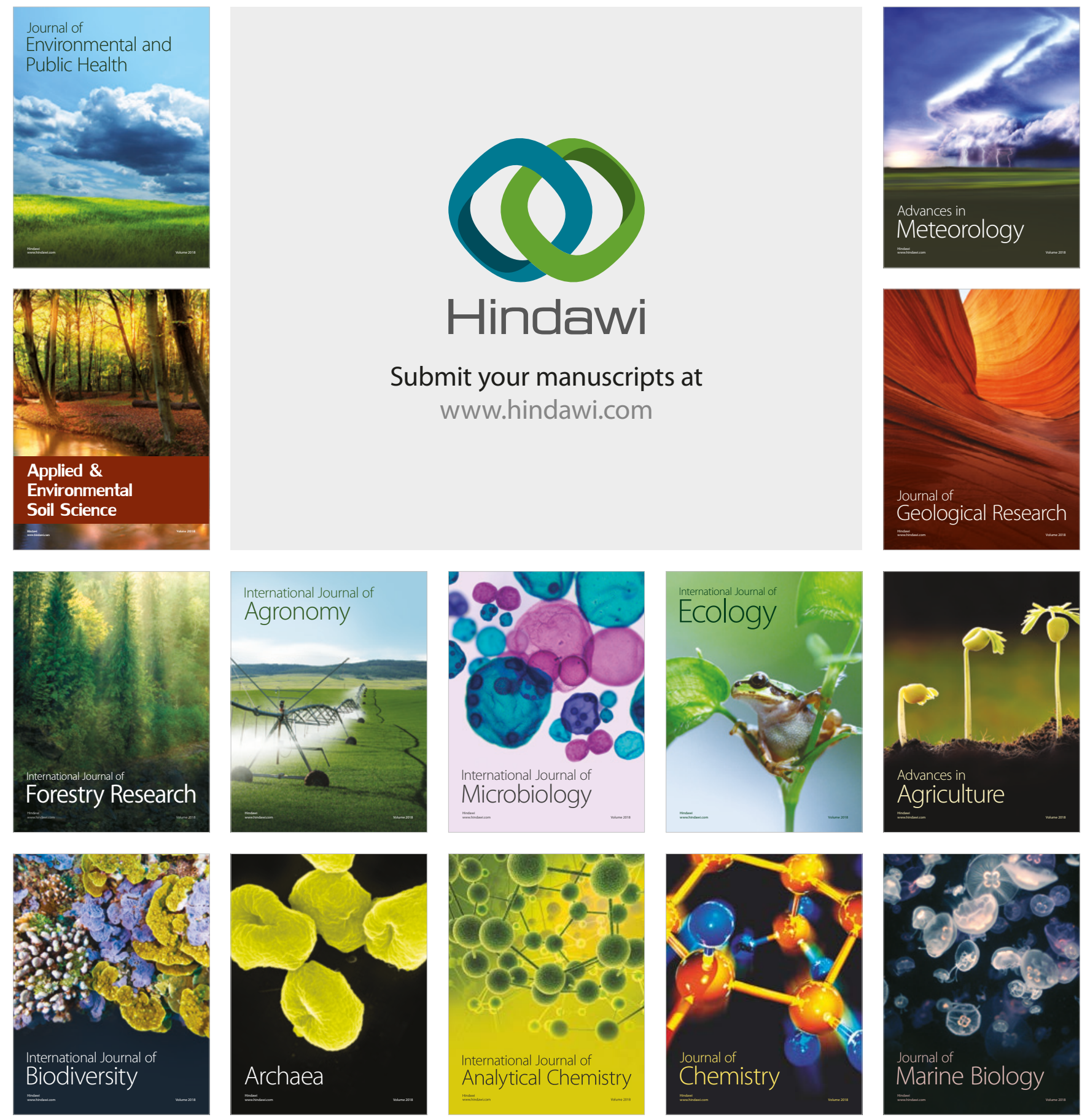